\title{
SPIRALLING IN PLANE RANDOM WALK
}

E. M. Wright*

(received June 10, 1964)

1. A particle is initially at the origin in the $(X, Y)$ plane and each successive step it takes is of unit length and paralleI either to the $\mathrm{X}$-axis or to the $\mathrm{Y}$-axis. Its path of $\mathrm{n}$ steps is called a spiral if (i) the particle never occupies the same position twice, (ii) any turns the path makes are all counter-clockwise or all clockwise and (iii) for every $m>n$, the path can be continued to $m$ steps without violating (i) or (ii). The condition (iii) is designed to exclude a path such as $(0,0)-(1,0)-(1,1)$ $-(1,2)-(0,2)-(0,1)$.

Let $s_{n}$ be the number of $n$-step spirals and $p(n)$ be the number of unrestricted partitions of $n$. In [2] Melzak used generating functions to prove that

$$
\mathrm{s}_{\mathrm{n}}=4\left\{1+2 \sum_{\mathrm{m}=1}^{\mathrm{n}-1} \mathrm{p}(\mathrm{m})\right\} .
$$

Here we give a direct "geometrical" proof of this result which makes (1) fairly transparent. We add a proof by generating functions, alternative to Melzak's, using a well-known identity due to Jacobi, and suggest an unsolved problem, which seems more difficult.

We call each successive straight part of the spiral a straight $i . e$. the set of steps in the same direction between the

The research reported herein has been sponsored by the Office, Chief of Research and Development, U.S. Department of the Army, through its European Research Office under contract DA- $91-591-$ EUC- 3256 .

Canad. Math. Bull. vol. 8, no. 1, February 1965 
origin and the first turn, between one turn and the next, between the last turn and the end, or between the origin and the end the last in the case in which the spiral has noturns). A spiral is characterised (i) by the direction in which its tail, i. e. its last straight, points, (ii) if it is not simply a single straight, by whether its turns are all counter-clockwise or all clockwise and (iii) by the finite sequence $b_{0}, b_{1}, \ldots$ of the number of steps in each of its $k+1$ straights, starting with the tail of $b_{0}$ steps. The $b$ must satisfy

$$
1 \leq \mathrm{b}_{0} \leq \mathrm{n}
$$

$$
\mathrm{b}_{1}>\mathrm{b}_{3}>\ldots>\mathrm{b}_{2 \mathrm{v}-1} \geq 1 ; \mathrm{b}_{2}>\mathrm{b}_{4}>\ldots>\mathrm{b}_{2 \mathrm{~V}} \geq 1
$$

where $\mathrm{V}=\mathrm{v}=\frac{1}{2} \mathrm{k}$ if $\mathrm{k}$ is even and $\mathrm{V}=\mathrm{v}-1=\frac{1}{2}(\mathrm{k}-1)$ if $\mathrm{k}$ is odd. Of these, (2) and (3) are obvious, while (4) expresses the essential spiral property, viz. that each straight (except the tail) must be longer than the next parallel straight.

We call a spiral whose tail points downwards (i.e. in the negative $Y$-axis direction) an $S^{\prime}$. An $S^{\prime}$ which has at least one counter-clockwise turn is called an $S^{\prime \prime}$. Let $S_{n}^{\prime}$ and $s_{n}^{\prime \prime}$ be the number of $n-s t e p S^{\prime}$ and $S^{\prime \prime}$ respectively.

There is an equal probability of the tail of a spiral pointing in any one of the four directions and so $s_{n}=4 s_{n}^{\prime}$. Again, $s_{n}^{\prime}$ enumerates one straight spiral and otherwise equal numbers of counter-clockwise and clockwise spirals. Hence

$$
s_{n}=4 s_{n}^{\prime}=4\left(1+2 s_{n}^{\prime \prime}\right)
$$

The spiral which we can obtain by removing the tail from an $S^{\prime \prime}$ we call a $T$. The successive straights of a $T$ (going inwards) contain $b_{1}, b_{2}, \ldots$ steps, where (4) is satisfied, Let $t(n)$ be the number of different $n$-step $T$. Since every $n$-step $S^{\prime \prime}$ has at least one turn, its tail has $b_{0}$ steps, where 


$$
1 \leq \mathrm{b}_{0} \leq \mathrm{n}-1
$$

and so

$$
s_{n}^{\prime \prime}=\sum_{b_{0}=1}^{n-1} t\left(n-b_{0}\right)=\sum_{m=1}^{n-1} t(m) .
$$

Hence, by (5) and (6). we have only to prove that

$$
t(n)=p(n)
$$

to have (1).

2. We now dissect an n-step $T$. The successive horizontal straights ( $v$ in number) containing $b_{1}, b_{3}, b_{5}, \ldots, b_{2 v-1}$ steps respectively, are placed under one another at unit distance, the left-hand end of each being one step to the right of the left-hand end of the one above. In view of the first part of (4), the righthand end of any straight does not project beyond the right-hand end of the one above. The vertical straights ( $V$ in number, where $\mathrm{V}=\mathrm{v}$ or $\mathrm{v}-1)$, containing $\mathrm{b}_{2}, \mathrm{~b}_{4}, \ldots, \mathrm{b}_{2 \mathrm{~V}}$ steps respectively, are arranged as shown in Figure 1. Again the bottom end of one of these straights cannot project below the bottom end of the one to its left. The left hand diagram in Figure 1 shows a case in which $\mathrm{V}=\mathrm{v}-1$, the right hand diagram a case in which $\mathrm{V}=\mathrm{v}$.

Now replace each horizontal step by its left-hand end-point and each vertical step by its lower end-point. The result is shown in Figure 2. We now have the usual node diagram of a partition of $n$. We observe that, whether $V=v$ or $v-1$, the Durfee square of the partition contains $v^{2}$ nodes.

The above process is unique and can obviously be reversed uniquely. Thus we have established a $(1,1)$ correspondence between the $n$-step $T$ and partitions of $n$. Hence ( 7 ).

This method is related to the ideas of [3]. 


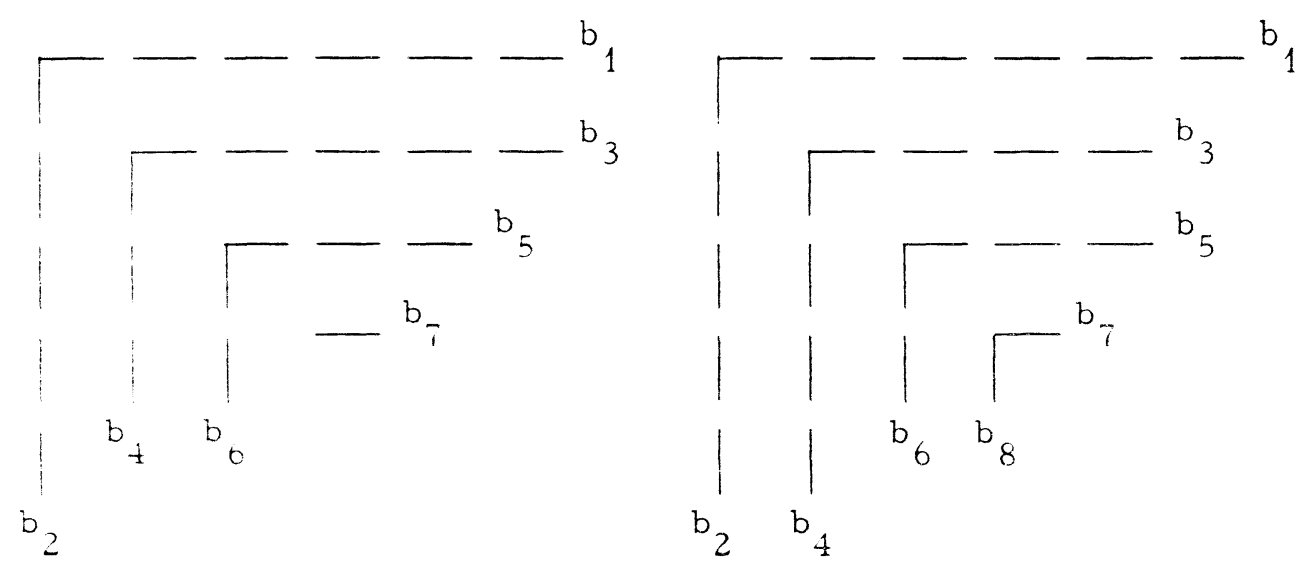

Figure 1

Figure 2 
3. As an alternative method, we write

$$
\prod_{r=1}^{\infty}\left(1+Z x^{r}\right)=\sum_{v=0}^{\infty} \sum_{n=0}^{\infty} M(v, n) Z^{v} X^{n}
$$

where $M(v, n)$ is the number of solutions of

$$
\mathrm{n}=\mathrm{b}_{1}+\mathrm{b}_{3}+\ldots+\mathrm{b}_{2 \mathrm{v}-1}\left(\mathrm{~b}_{1}>\mathrm{b}_{3}>\ldots \mathrm{b}_{2 \mathrm{v}-1}>0\right)
$$

for the particular $v$ in question. Now $t(n)$ is the number of solutions of

$$
\mathrm{n}=\mathrm{b}_{1}+\mathrm{b}_{2}+\ldots+\mathrm{b}_{\mathrm{v}}+\mathrm{V}
$$

satisfying (4), where $\mathrm{v}$ takes any positive value and $\mathrm{V}=\mathrm{v}-1$ or v. Hence

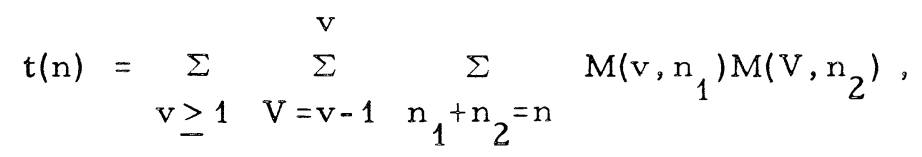

which is the coefficient of $z^{0} x^{n}$ in

$$
\Lambda=\Lambda(Z, X)=(1+Z) \prod_{r=1}^{\infty}\left(1+Z X^{r}\right)\left(1+Z^{-1} X^{r}\right)
$$

Hence $t(n)$ is the coefficient of $W^{0} Y^{2 n}$ in $\Lambda\left(W Y, Y^{2}\right)$. By Jaccbi's well-known identity (Theorem 352 of [1]), we see that

$$
\begin{aligned}
& \Lambda\left(W Y, Y^{2}\right)=\prod_{r=1}^{\infty}\left\{\left(1+W Y^{2 r-1}\right)\left(1+W^{-1} Y^{2 r-1}\right)\right\} \\
& =\sum_{\mathrm{k}=-\infty}^{\infty} \mathrm{W}^{\mathrm{k}} \mathrm{Y}^{\mathrm{k}^{2}} \prod_{\mathrm{s}=1}^{\infty}\left(1-\mathrm{Y}^{2 \mathrm{~s}}\right)^{-1}
\end{aligned}
$$

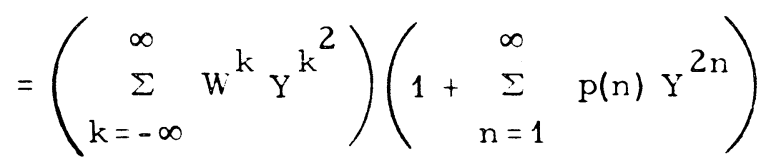


4. The next stage of complexity is to consider random walks on a lattice consisting of congruent equilateral triangles. It can be shown that, in this case, the number $s_{n}$ of $n$-step spirals is

$$
s_{n}=6\left\{1+2 \sum_{m=1}^{n-1} t(m)\right\}
$$

where $t(n)$ is the number of solutions in non-negative integers of the equation

$$
n=a_{1}+a_{2}+\ldots+a_{k}
$$

for any $k$ such that

$$
\begin{aligned}
& 0<a_{1}<a_{3}+a_{4}<a_{6}+a_{7}<\ldots \\
& 0<a_{1}+a_{2}<a_{4}+a_{5}<a_{7}+a_{8}<\ldots \\
& 0<a_{2}+a_{3}<a_{5}+a_{6}<a_{8}+a_{9}<\ldots .
\end{aligned}
$$

But I cannot relate this $t(n)$ to any known partition function. It would be interesting if this were possible.

\section{REFERENCES}

1. G. H. Hardy and E. M. Wright, Theory of Numbers, 4th edition, Oxford (1960).

2. Z.A. Melzak, Partition Functions and Spiralling in Plane Random Walk, Can. Math. Bull. 6(1963), 231-237.

3. E.M. Wright, An enumerative proof of an identity of Jacobi, Journ. London Math. Soc. 40(1965), 55-57.

University of Aberdeen, Scotland 